\title{
FUZZY ANALYSIS FOR ASSESSMENT OF RIVER WATER QUALITY
}

\author{
Sonal Bhugra ${ }^{1}$, Sria Biswas ${ }^{2}$ \\ ${ }^{1}$ Assistant Professor, Deptt. of Civil Engg., Manav Rachna International University, Faridabad, India \\ ${ }^{2}$ M.Tech Student, Dept. of Nano Technology, Jadavpur University, Kolkata, India,
}

\begin{abstract}
River water gets polluted due to rapid industrialization and urbanization with inadequate cognizance to the implementation of pollution control measures. Due to asymmetric occurrence of pollutants at various locations of the river the environmental impact assessment (EIA) has an important role in defining river water quality. At present experts are facing lots of problems to decide for a proposed development project based on the laboratory test results only. Because no tool are available to them which can give precise information or knowledge for taking best decision in favour of the project except the process of EIA[4,6]. But in EIA, most of the parametric information or data are not always crisp or precise rather linguistic and hedges viz. "good discharge", "less turbidity", "high pH”, "low BOD”, etc. to list a few only out of infinity. All such type of data are fuzzy in nature naturally evaluation of such fuzzy data is not possible with numerical valued description. As human beings every decision-maker hesitates more or less on every evaluation activity because some part of his evaluation contributes to truthness, some part to falseness and rest part may be indeterministic to him[3]. So uncertainty is the great problems to an experts while taking any decision. In this paper a methodology of Fuzzy EIA has been studied using the logic of Intuitionistic Fuzzy Set (IFS)[1] theory for assessment of river water quality with degree of certainty.
\end{abstract}

Keywords: fuzzy-EIA, IFS, mean fuzzy set, uncertainty, union fuzzy set, etc.

\section{INTRODUCTION}

The continuous pollution resulting from various sources deteriorates the fresh water quality and affects its quantity. To protect the river from anticipated environmental degradation, an EIA has the vital role to a water quality management authority. EIA leads a systematic analysis using information collected from different sources and then predict the environmental consequences of any human development project[5]. So EIA is the prediction process thus uncertainty is an integral part of it. Generally there are two types of uncertainty associated in EIA : that associated with the process and, that associated with predictions. The accuracy of predictions is dependent on a variety of factors such as lack of precise data or lack of precise knowledge [3]. So considerable uncertainty and impreciseness are involved in the process of defining river water quality which could be solved using powerful mathematical tool of fuzzy logic.

Prof. Latfi Zadeh, first laid the foundation of fuzzy logic i.e fuzzy set theory in 1965 as a modification of ordinary crisp set theory. It provides a formal process for representing and reasoning with uncertainty information involved in the linguistic variable[3]. At present day there is a tremendous application of fuzzy logic in different technical field because of the fact that it has the capability to handle the linguistic variable in terms of numerical data. According to concept of fuzzy logic, When a statement is completely true then the membership value is 1 and when a statement is completely false the membership value is 0 and when the statement is partly true or partly false then the membership value will be in between 0 and 1. Thus the membership value of any element of fuzzy set can take any value form the closed interval $[0,1]$ in such a condition that sum of membership value and non membership value should always be one[7]. But in few cases of judgment when hesitation plays a major role in evaluation of uncertainty then only simple fuzzy logic of Prof. Zadeh do not give the better result. To eliminate this hesitation part of the uncertainty, Prof. K.T.Atanasove introduced further a new higher order concept of Instuitionistic Fuzzy Set (IFS) theory[1]. He bifurcated the non membership value of Prof Zadeh into two parts : one is purely non membership and other is indeterministic part or hesitation part. According to his logic, some part of the evaluation contributes truthness, some part of the evaluation falseness and rest part is hesitation. When this hesitation part will be zero then it will act as an ordinary fuzzy logic of Prof. Zadeh. This is called the breaking philosophy of Prof. K.T.Atanasove[1]. In this paper the logic of Intuitionistic Fuzzy Set (IFS) theory is used in modeling of Fuzzy-EIA to tackle the uncertainty in defining river water quality more precisely.

\section{METHODOLOGY OF 'FUZZY EIA'}

To understand the methodology of Fuzzy-EIA, few definitions are presented below

\section{Definition 2.1 Intuitionistic Fuzzy Set (IFS)}

An Intuitionistic Fuzzy Set (IFS) A in E is defined as

$\mathrm{A}=\left\{\left(\mathrm{x}, \mu_{\mathrm{A}}(\mathrm{x}), \mathrm{v}_{\mathrm{A}}(\mathrm{x})\right) \mid \mathrm{x} \in \mathrm{E}\right\}$, where $\mu_{\mathrm{A}}(\mathrm{x})+\mathrm{v}_{\mathrm{A}}$ $(\mathrm{x})+\pi_{\mathrm{A}}(\mathrm{x})=1$

and for every $\mathrm{x} \in \mathrm{E}$ there is a condition that $0 \leq \mu_{\mathrm{A}}(\mathrm{x})+$ $\mathrm{v}_{\mathrm{A}}(\mathrm{x}) \leq 1$. 


\section{Definition 2.2 Attributes of the Assessment}

In EIA information or data are collected for evaluation of certain attributes which are called the attributes of the assessment. For example, consider a project 'ASSESSMENT OF RIVER WATER QUALITY', for which some relevant attributes could be "good discharge", "less turbidity", "high pH", "low BOD”, "high alkalinity", etc.

\section{Definition 2.2 Universe of the Assessment}

Collection of all attributes of the assessment is called the Universe of the Assessment.

Let $E=\left\{x_{1}, x_{2} \ldots \ldots \ldots, x_{n}\right\}$ be a finite discrete universe of attributes $\mathrm{x}_{\mathrm{i}}, i=1,2, . ., \mathrm{n}$.

\section{Definition 2.4 Mean Fuzzy Set of IFS}

Let $\mathrm{E}$ be an universe and $\mathrm{A}$ be an IFS of $\mathrm{E}$. The mean fuzzy set of IFS A is a fuzzy set $B$ of $E$ given by the membership function such that

$$
\mu(x)_{B}=\frac{\mu_{A}(x)+1-v_{A}(x)}{2}
$$

\section{Definition 2.3 Union of Mean Fuzzy Sets}

If $A$ and $B$ are two mean fuzzy sets of universe $U$, then union of mean fuzzy sets A and B will be a fuzzy set $X$ with the membership function $\mu_{A \cup B}$. Example : If $A$ and $B$ are two mean fuzzy sets such that

$\mathrm{A}=\{\mathrm{a} / 0.4, \mathrm{~b} / 0.2, \mathrm{c} / 0.5, \mathrm{~d} / 0.5\}$ and $\mathrm{B}=\{\mathrm{a} / 0.2, \mathrm{~b} / 0.9$, $\mathrm{c} / 0.7, \mathrm{~d} / 0.5\}$, then union fuzzy set $\mathrm{X}$ of mean fuzzy sets $\mathrm{A}$ and $B$ will be $X\left(\mu_{A \cup B}\right)=\{a / 0.4, b / 0.9, c / 0.7, d / 0.5\}$.

\section{Definition 2.5 Weighted Average of an Union Fuzzy}

\section{Set}

Let $X$ be an union fuzzy set with membership function $\mu(\mathrm{x})$ of universe E. Suppose that to each element $x \in E$, there is an associated weight $\mathrm{W}_{\mathrm{x}} \in R+$ (set of all non-negative real numbers). Then the weighted average of the fuzzy set $X$ is the non-negative number a $(\mathrm{X})$ given by

$$
a(X)=\quad \frac{\sum \mu(x) \cdot W_{x}}{\sum W_{x}}
$$

\section{Definition 2.6 Grading of Assessment Output}

In Fuzzy-EIA modeling, evaluation of all attributes are done either based on their negative aspects(draw backs) or positive aspects. If negative aspects of all attributes are consider then grading of output results of Fuzzy-EIA could be proposed as below:

$$
\begin{array}{lll}
\text { grade }=\text { Not acceptable if } & .8<\mathrm{a}(\mathrm{X}) \leq 1 \\
\text { grade }=\text { Just acceptable if } & .6<\mathrm{a}(\mathrm{X}) \leq .8 \\
\text { grade }=\text { Acceptable } & \text { if } & .4<\mathrm{a}(\mathrm{X}) \leq .6 \\
\text { grade }=\text { Moderately acceptable } & \text { if } .2<\mathrm{a}(\mathrm{X}) \leq .4 \\
\text { grade }=\text { Highly acceptable if } & 0 \leq \mathrm{a}(\mathrm{X}) \leq .2
\end{array}
$$

Similarly for positive aspects, grading of output result of Fuzzy-EIA could be proposed as:

$$
\begin{array}{lrlrl}
\text { grade } & =\text { Highly acceptable } & \text { if } & .8 & <\mathrm{a}(\mathrm{X}) \leq 1 \\
\text { grade }=\text { Moderately acceptable } & \text { if } .6 & <\mathrm{a}(\mathrm{X}) \leq .8 \\
\text { grade }=\text { Acceptable } & \text { if } & .4 & <\mathrm{a}(\mathrm{X}) \leq .6 \\
\text { grade }=\text { Just acceptable } & & \text { if } .2<\mathrm{a}(\mathrm{X}) \leq .4 \\
\text { grade }=\text { Not acceptable } & \text { if } & 0 \leq \mathrm{a}(\mathrm{X}) \leq .2
\end{array}
$$

Now to validate the model of Fuzzy-EIA a case study is presented below.

\begin{tabular}{|c|c|c|c|c|c|c|c|c|c|c|}
\hline Parameters & $\mathbf{L}_{1}$ & $\mathbf{L}_{2}$ & $\mathbf{L}_{3}$ & $\mathbf{L}_{4}$ & $\mathbf{L}_{5}$ & $L_{6}$ & $\mathbf{L}_{7}$ & $\mathbf{L}_{8}$ & $\mathbf{L}_{9}$ & $\mathrm{~L}_{\mathbf{1 0}}$ \\
\hline $\mathrm{PH}$ & $\begin{array}{l}7.85- \\
8.90\end{array}$ & $\begin{array}{l}8.05 \\
9.25\end{array}$ & $\begin{array}{l}7.85 \\
9.40\end{array}$ & $\begin{array}{l}7.41- \\
9.00\end{array}$ & $\begin{array}{l}7.36 \\
8.98\end{array}$ & $\begin{array}{l}7.40 \\
9.2\end{array}$ & $\begin{array}{l}7.20- \\
9.20\end{array}$ & $\begin{array}{l}7.10 \\
9.30\end{array}$ & $\begin{array}{l}7.30 \\
9.10\end{array}$ & $\begin{array}{l}7.32 \\
9.00\end{array}$ \\
\hline $\begin{array}{l}\text { Turbidity } \\
\text { (NTU) }\end{array}$ & $\begin{array}{l}14- \\
1390\end{array}$ & $\begin{array}{ll}28 & - \\
2700 & \\
\end{array}$ & $\begin{array}{ll}64 & - \\
3300 & \end{array}$ & $\begin{array}{l}79 \\
4100 \\
\end{array}$ & $\begin{array}{l}76 \\
4000 \\
\end{array}$ & $\begin{array}{ll}75 & - \\
4200 & \\
\end{array}$ & $\begin{array}{l}73- \\
4400\end{array}$ & $\begin{array}{l}88 \\
3900 \\
\end{array}$ & $\begin{array}{l}83 \\
4150 \\
\end{array}$ & $\begin{array}{l}77 \\
4500 \\
\end{array}$ \\
\hline $\begin{array}{l}\text { Alkalinity } \\
(\mathrm{mg} / \mathrm{l})\end{array}$ & $\begin{array}{ll}26 & - \\
96 & \\
\end{array}$ & $28-121$ & $26-110$ & $24-199$ & $20-168$ & $26-130$ & $\begin{array}{l}28- \\
227 \\
\end{array}$ & $22-237$ & $39-235$ & $32-210$ \\
\hline $\begin{array}{l}\text { Hardness } \\
\text { as }\left(\mathrm{CaCO}_{3}\right)\end{array}$ & $\begin{array}{ll}19 & - \\
99 & \end{array}$ & $25-108$ & $20-96$ & $25-103$ & $33-98$ & $28-92$ & $28-96$ & $32-110$ & $22-117$ & $18-105$ \\
\hline
\end{tabular}

\section{CASE STUDY}

Consider a project "ASSESSMENT OF RIVER WATER QUALITY " . For case study we select the river 'HOWRAH' of Tripura State, India. For evaluation of fizzy EIA, we select ten locations of the river and ten individual water quality experts for each location. The laboratory test results of water samples of those ten locations are collected from the sources of PHE Department, Govt. of Tripura and presented in the form of interval data in table no-1. We consider here only last three years test results as a basic data of this case study.

Table: 1 


\begin{tabular}{|c|c|c|c|c|c|c|c|c|c|c|}
\hline $\mathrm{TS}(\mathrm{mg} / \mathrm{l})$ & $\begin{array}{l}415- \\
8900 \\
\end{array}$ & $\begin{array}{l}390 \quad- \\
15000\end{array}$ & $\begin{array}{l}470 \\
16300\end{array}$ & $\begin{array}{l}490 \\
21000\end{array}$ & $\begin{array}{ll}510 & - \\
20800 & \\
\end{array}$ & $\begin{array}{l}510- \\
23400\end{array}$ & $\begin{array}{l}395- \\
22900 \\
\end{array}$ & $\begin{array}{l}530- \\
24100\end{array}$ & $\begin{array}{l}415- \\
22400\end{array}$ & $\begin{array}{l}490 \\
23100\end{array}$ \\
\hline TDS (mg/l) & $\begin{array}{l}106- \\
2700\end{array}$ & $\begin{array}{ll}110 & - \\
5100 & \end{array}$ & $\begin{array}{l}106 \\
5800\end{array}$ & $\begin{array}{ll}88 & - \\
6900 & \end{array}$ & $\begin{array}{ll}98 & - \\
7200 & \end{array}$ & $\begin{array}{ll}105 & - \\
7100 & \end{array}$ & $\begin{array}{l}100- \\
6800\end{array}$ & $\begin{array}{ll}115 & - \\
8000 & \end{array}$ & $\begin{array}{ll}104 & - \\
7899 & \end{array}$ & $\begin{array}{ll}98 & - \\
8200 & \end{array}$ \\
\hline $\begin{array}{l}\text { Chloride } \\
(\mathrm{mg} / \mathrm{l})\end{array}$ & $\begin{array}{l}4.78- \\
32\end{array}$ & $4.78-25$ & $10-28$ & $9-32$ & $11-30$ & $8-28$ & $10-30$ & $13-27$ & $8-30$ & $9-27$ \\
\hline Iron $(\mathrm{mg} / \mathrm{l})$ & $\begin{array}{l}1.2- \\
3.90\end{array}$ & $\begin{array}{ll}1.0 & - \\
4.10 & \end{array}$ & $\begin{array}{l}1.3 \\
4.00\end{array}$ & $\begin{array}{l}1.1 \\
4.80\end{array}$ & $\begin{array}{ll}1.4 & - \\
4.450 & \end{array}$ & $\begin{array}{l}1.0 \\
4.75\end{array}$ & $\begin{array}{l}1.10- \\
4.50\end{array}$ & $\begin{array}{l}1.50 \\
4.20\end{array}$ & $\begin{array}{l}1.2 \\
4.00\end{array}$ & $\begin{array}{l}1.0 \\
4.50\end{array}$ \\
\hline $\begin{array}{l}\text { Total } \\
\text { coliform } \\
\text { (MPN/100ml } \\
\text { ) }\end{array}$ & $\begin{array}{l}210- \\
1630\end{array}$ & $\begin{array}{ll}350 & - \\
1790 & \end{array}$ & $\begin{array}{l}210 \\
1680\end{array}$ & $\begin{array}{l}460 \\
1930\end{array}$ & $\begin{array}{l}750 \\
1850\end{array}$ & $\begin{array}{l}630 \\
1890\end{array}$ & $\begin{array}{l}610- \\
1850\end{array}$ & $\begin{array}{l}710 \\
1900\end{array}$ & $\begin{array}{l}685 \\
1800\end{array}$ & $\begin{array}{l}900 \\
1850\end{array}$ \\
\hline DO (mg/l) & $\begin{array}{l}3.13- \\
9.75\end{array}$ & $\begin{array}{l}4.25- \\
7.98\end{array}$ & $\begin{array}{l}3.70- \\
8.90\end{array}$ & $\begin{array}{l}4.60- \\
9.89\end{array}$ & $\begin{array}{l}3.85- \\
8.86\end{array}$ & $\begin{array}{l}4,75- \\
7.95\end{array}$ & $\begin{array}{l}5.60- \\
9.80\end{array}$ & $\begin{array}{l}4.39- \\
8.90\end{array}$ & $\begin{array}{l}3.20- \\
9.70\end{array}$ & $\begin{array}{l}5.10- \\
9.20 .\end{array}$ \\
\hline
\end{tabular}

In Fuzzy EIA modeling, attributes are the main keys for evaluation and for simplicity in presenting it's methodology, we consider the following ten attributes (Drawbacks) for each location of the river :-

$\begin{array}{lll}\mathrm{x}_{1}= & & \text { high turbidity } \\ \mathrm{x}_{2} & = & \text { high alkalinity } \\ \mathrm{x}_{3} & = & \text { high } \mathrm{pH} \\ \mathrm{x}_{4} & = & \text { high Nitrate } \\ \mathrm{x}_{5} & = & \text { high DO } \\ \mathrm{x}_{6} & = & \text { high BOD } \\ \mathrm{x}_{7} & = & \text { high COD } \\ \mathrm{x}_{8} & = & \text { high E-coli (MPN) } \\ \mathrm{x}_{9} & = & \text { high arsenic } \\ \mathrm{x}_{10} & = & \text { high chloride }\end{array}$

Naturally, all independent expert's views for individual locations will lead to an individual IFS of the universe $\mathrm{U}$,

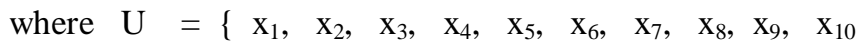
\} .

Suppose all individual IFS are designated as $\mathrm{L}_{1}, \mathrm{~L}_{2}, \mathrm{~L}_{3}, \mathrm{~L}_{4}$, $\mathrm{L}_{5}, \quad \mathrm{~L}_{6}, \mathrm{~L}_{7}, \mathrm{~L}_{8}, \mathrm{~L}_{9}, \mathrm{~L}_{10}$ and the membership functions generated from the expert's views for each IFS are described as :

$\mathrm{L}_{1}=\left\{\left(\mathrm{x}_{1}, .70, .20\right),\left(\mathrm{x}_{2}, .65, .15\right),\left(\mathrm{x}_{3}, .60, .30\right),\left(\mathrm{x}_{4}, .60\right.\right.$, $.30),\left(\mathrm{x}_{5}, .45, .35\right),\left(\mathrm{x}_{6}, .20, .60\right),\left(\mathrm{x}_{7}, .40, .50\right),\left(\mathrm{x}_{8}, .20, .70\right)$, $\left.\left(\mathrm{x}_{9}, .85, .10\right),\left(\mathrm{x}_{10}, .55, .25\right)\right\}$.

$\mathrm{L}_{2}=\left\{\left(\mathrm{x}_{1}, .75, .20\right),\left(\mathrm{x}_{2}, .60, .25\right),\left(\mathrm{x}_{3}, .40, .60\right),\left(\mathrm{x}_{4}\right.\right.$, $.30, .70),\left(\mathrm{x}_{5}, .65,20\right),\left(\mathrm{x}_{6}, .65 .20\right),\left(\mathrm{x}_{7}, .30, .55\right),\left(\mathrm{x}_{8}, .55\right.$, $\left..20),\left(x_{9}, .20, .60\right),\left(x_{10}, .80, .15\right)\right\}$.

$\mathrm{L}_{3}=\left\{\left(\mathrm{x}_{1}, .40, .45\right),\left(\mathrm{x}_{2}, .40, .55\right),\left(\mathrm{x}_{3}, .70, .25\right),\left(\mathrm{x}_{4}, .55\right.\right.$, $.30),\left(\mathrm{x}_{5}, .70, .10\right),\left(\mathrm{x}_{6}, .75, .10\right),\left(\mathrm{x}_{7}, .80, .10\right),\left(\mathrm{x}_{8}, .50, .25\right)$, $\left.\left(\mathrm{x}_{9}, .60, .30\right),\left(\mathrm{x}_{10}, .65, .10\right)\right\}$.

$\mathrm{L}_{4}=\left\{\left(\mathrm{x}_{1}, .10, .70\right),\left(\mathrm{x}_{2}, .30, .55\right),\left(\mathrm{x}_{3}, .10, .80\right),\left(\mathrm{x}_{4}, .25\right.\right.$, $.70),\left(\mathrm{x}_{5}, .35, .55\right),\left(\mathrm{x}_{6}, .65 .25\right),\left(\mathrm{x}_{7}, .45, .50\right),\left(\mathrm{x}_{8}, .30, .60\right)$, $\left.\left(\mathrm{x}_{9}, .30, .50\right),\left(\mathrm{x}_{10}, .20, .65\right)\right\}$.
$\mathrm{L}_{5}=\left\{\left(\mathrm{x}_{1}, .25, .55\right),\left(\mathrm{x}_{2}, .80, .15\right),\left(\mathrm{x}_{3}, .80, .10\right),\left(\mathrm{x}_{4}, .50\right.\right.$, $.25),\left(\mathrm{x}_{5}, .75, .15\right),\left(\mathrm{x}_{6}, .80, .15\right),\left(\mathrm{x}_{7}, .60, .25\right),\left(\mathrm{x}_{8}, .50, .35\right)$, $\left.\left(\mathrm{x}_{9}, .50, .45\right),\left(\mathrm{x}_{10}, .85, .10\right)\right\}$.

$\mathrm{L}_{6}=\left\{\left(\mathrm{x}_{1}, .55, .20\right),\left(\mathrm{x}_{2}, .50, .45\right),\left(\mathrm{x}_{3}, .70, .20\right),\left(\mathrm{x}_{4}, .60\right.\right.$, $.30),\left(\mathrm{x}_{5}, .45, .50\right),\left(\mathrm{x}_{6}, .20 .75\right),\left(\mathrm{x}_{7}, .65, .30\right),\left(\mathrm{x}_{8}, .80, .15\right)$, $\left.\left(\mathrm{x}_{9}, .35, .55\right),\left(\mathrm{x}_{10}, .45, .30\right)\right\}$.

$\mathrm{L}_{7}=\left\{\left(\mathrm{x}_{1}, .20, .70\right),\left(\mathrm{x}_{2}, .20, .70\right),\left(\mathrm{x}_{3}, 80, .10\right),\left(\mathrm{x}_{4}, .85\right.\right.$ $.10),\left(\mathrm{x}_{5}, .55, .10\right),\left(\mathrm{x}_{6}, .50, .45\right),\left(\mathrm{x}_{7}, .40, .55\right),\left(\mathrm{x}_{8}, . .60, .25\right)$, $\left.\left(\mathrm{x}_{9}, .80, .15\right),\left(\mathrm{x}_{10}, .65, .15\right)\right\}$.

$\mathrm{L}_{8}=\left\{\left(\mathrm{x}_{1}, .60, .20\right),\left(\mathrm{x}_{2}, .85, .10\right),\left(\mathrm{x}_{3}, .45, .45\right),\left(\mathrm{x}_{4}, .70\right.\right.$, $.25),\left(\mathrm{x}_{5}, .75, .20\right),\left(\mathrm{x}_{6}, .70, .20\right),\left(\mathrm{x}_{7}, .70, .25\right),\left(\mathrm{x}_{8}, .50, .30\right)$, $\left.\left(\mathrm{x}_{9}, .65, .25\right),\left(\mathrm{x}_{10}, .70, .15\right)\right\}$.

$\mathrm{L}_{9}=\left\{\left(\mathrm{x}_{1}, .35, .60\right),\left(\mathrm{x}_{2}, 55, .35\right),\left(\mathrm{x}_{3}, .50, .20\right),\left(\mathrm{x}_{4}, .65\right.\right.$, $25),\left(\mathrm{x}_{5}, .70, .15\right),\left(\mathrm{x}_{6}, .60, .35\right),\left(\mathrm{x}_{7}, .35, .60\right),\left(\mathrm{x}_{8}, .40, .25\right)$, $\left.\left(\mathrm{x}_{9}, .70, .20\right),\left(\mathrm{x}_{10}, .60, .30\right)\right\}$.

$\mathrm{L}_{10}=\left\{\left(\mathrm{x}_{1}, .70, .20\right),\left(\mathrm{x}_{2}, .75, .15\right),\left(\mathrm{x}_{3}, .85, .10\right),\left(\mathrm{x}_{4}, .90\right.\right.$, $.05),\left(\mathrm{x}_{5}, .65, .30\right),\left(\mathrm{x}_{6}, .75, .15\right),\left(\mathrm{x}_{7}, .85, .10\right),\left(\mathrm{x}_{8}, .65, .25\right)$, $\left.\left(\mathrm{x}_{9}, .45, .40\right),\left(\mathrm{x}_{10}, .55, .35\right)\right\}$.

Now our job is to calculate the mean fuzzy sets of each which could be as :

$\mathrm{L}_{1}=\left\{\left(\mathrm{x}_{1}, .75\right),\left(\mathrm{x}_{2}, .75\right),\left(\mathrm{x}_{3}, .65\right),\left(\mathrm{x}_{4}, .65\right),\left(\mathrm{x}_{5}, .55\right),\left(\mathrm{x}_{6}\right.\right.$, $\left..30),\left(\mathrm{x}_{7}, .45\right),\left(\mathrm{x}_{8}, .25\right),\left(\mathrm{x}_{9}, .875\right),\left(\mathrm{x}_{10}, .65\right)\right\}$.

$\mathrm{L}_{2}=\left\{\left(\mathrm{x}_{1}, .775\right),\left(\mathrm{x}_{2}, .675\right),\left(\mathrm{x}_{3}, .40\right),\left(\mathrm{x}_{4}, .30\right),\left(\mathrm{x}_{5}, .725\right)\right.$, $\left.\left(\mathrm{x}_{6}, .725\right),\left(\mathrm{x}_{7}, .375\right),\left(\mathrm{x}_{8}, .675\right),\left(\mathrm{x}_{9}, .30\right),\left(\mathrm{x}_{10}, .825\right)\right\}$.

$\mathrm{L}_{3}=\left\{\left(\mathrm{x}_{1}, .475\right),\left(\mathrm{x}_{2}, .425\right),\left(\mathrm{x}_{3}, .725\right),\left(\mathrm{x}_{4}, .625\right),\left(\mathrm{x}_{5}, .80\right)\right.$, $\left.\left(\mathrm{x}_{6}, .825\right),\left(\mathrm{x}_{7}, .85\right),\left(\mathrm{x}_{8}, .625\right),\left(\mathrm{x}_{9}, .65\right),\left(\mathrm{x}_{10}, .775\right)\right\}$.

$\mathrm{L}_{4}=\left\{\left(\mathrm{x}_{1}, .20\right),\left(\mathrm{x}_{2}, .375\right),\left(\mathrm{x}_{3}, .15\right),\left(\mathrm{x}_{4}, .275\right),\left(\mathrm{x}_{5}, .40\right)\right.$, $\left.\left(\mathrm{x}_{6}, .70\right),\left(\mathrm{x}_{7}, .475\right),\left(\mathrm{x}_{8}, .35\right),\left(\mathrm{x}_{9}, .40\right),\left(\mathrm{x}_{10}, .275\right)\right\}$.

$\mathrm{L}_{5}=\left\{\left(\mathrm{x}_{1}, .35\right),\left(\mathrm{x}_{2}, .825\right),\left(\mathrm{x}_{3}, .85\right),\left(\mathrm{x}_{4}, .625\right),\left(\mathrm{x}_{5}, .80\right)\right.$, $\left.\left(\mathrm{x}_{6}, .825\right),\left(\mathrm{x}_{7}, .675\right),\left(\mathrm{x}_{8}, .575\right),\left(\mathrm{x}_{9}, .525\right),\left(\mathrm{x}_{10}, .875\right)\right\}$.

$\mathrm{L}_{6}=\left\{\left(\mathrm{x}_{1}, .675\right),\left(\mathrm{x}_{2}, .525\right),\left(\mathrm{x}_{3}, .75\right),\left(\mathrm{x}_{4}, .65\right),\left(\mathrm{x}_{5}, .475\right)\right.$, $\left.\left(\mathrm{x}_{6}, .225\right),\left(\mathrm{x}_{7}, .675\right),\left(\mathrm{x}_{8}, .825\right),\left(\mathrm{x}_{9}, .40\right),\left(\mathrm{x}_{10}, .575\right)\right\}$. 
$\mathrm{L}_{7}=\left\{\left(\mathrm{x}_{1}, .25\right),\left(\mathrm{x}_{2}, .25\right),\left(\mathrm{x}_{3}, 85\right),\left(\mathrm{x}_{4}, .875\right),\left(\mathrm{x}_{5}, .725\right),\left(\mathrm{x}_{6}\right.\right.$, $\left..525),\left(\mathrm{x}_{7}, .425\right),\left(\mathrm{x}_{8}, . .675\right),\left(\mathrm{x}_{9}, .825\right),\left(\mathrm{x}_{10}, .75\right)\right\}$.

$\mathrm{L}_{8}=\left\{\left(\mathrm{x}_{1}, .70\right),\left(\mathrm{x}_{2}, .875\right),\left(\mathrm{x}_{3}, .50\right),\left(\mathrm{x}_{4}, .725\right),\left(\mathrm{x}_{5}, .775\right)\right.$, $\left.\left(\mathrm{x}_{6}, .75\right),\left(\mathrm{x}_{7}, .725\right),\left(\mathrm{x}_{8}, .60\right),\left(\mathrm{x}_{9}, .70\right),\left(\mathrm{x}_{10}, .775\right)\right\}$.

$\mathrm{L}_{9}=\left\{\left(\mathrm{x}_{1}, .375\right),\left(\mathrm{x}_{2}, 60\right),\left(\mathrm{x}_{3}, .65\right),\left(\mathrm{x}_{4}, .70\right),\left(\mathrm{x}_{5}, .775\right),\left(\mathrm{x}_{6}\right.\right.$, $\left..625),\left(x_{7}, .375\right),\left(x_{8}, .575\right),\left(x_{9}, .75\right),\left(x_{10}, .65\right)\right\}$.

$\mathrm{L}_{10}=\left\{\left(\mathrm{x}_{1}, .75\right),\left(\mathrm{x}_{2}, .80\right),\left(\mathrm{x}_{3}, .875\right),\left(\mathrm{x}_{4}, .925\right),\left(\mathrm{x}_{5}, .675\right)\right.$, $\left.\left(\mathrm{x}_{6}, .80\right),\left(\mathrm{x}_{7}, .875\right),\left(\mathrm{x}_{8}, .70\right),\left(\mathrm{x}_{9}, .525\right),\left(\mathrm{x}_{10}, .60\right)\right\}$.

For overall assessment of water quality of river-HOWRAH, the union of all above fuzzy sets (as we consider the draw back of the attributes) will give the overall membership value of each attributes for the river. Thus the union fuzzy set $\mathrm{X}$ of the fuzzy sets $\mathrm{L}_{1}, \mathrm{~L}_{2}, \mathrm{~L}_{3}, \mathrm{~L}_{4}, \mathrm{~L}_{5}, \mathrm{~L}_{6}, \mathrm{~L}_{7}, \mathrm{~L}_{8}, \mathrm{~L}_{9}$, $\mathrm{L}_{10}$ will be

$\mathrm{X}=\mathrm{L}_{1} \cup \mathrm{L}_{2} \cup \mathrm{L}_{3} \cup \mathrm{L}_{4} \cup \mathrm{L}_{5} \cup \mathrm{L}_{6} \cup \mathrm{L}_{7} \cup \mathrm{L}_{8} \cup \mathrm{L}_{9} \cup \mathrm{L}_{10}$ $=\left\{\left(\mathrm{x}_{1}, .775\right),\left(\mathrm{x}_{2}, .875\right),\left(\mathrm{x}_{3}, .875\right),\left(\mathrm{x}_{4}, .925\right),\left(\mathrm{x}_{5}, .80\right),\left(\mathrm{x}_{6}\right.\right.$, $.825),\left(\mathrm{x}_{7}, .875\right),\left(\mathrm{x}_{8}, .825\right)$,

$\left.\left(\mathrm{x}_{9}, .875\right),\left(\mathrm{x}_{10}, .875\right)\right\}$.

Suppose the prefixed weigh of each attribute are consider as for $x_{1}=50$, for $x_{2}=60$, for $x_{3}=30$, for $x_{4}=40$, for $x_{5}=15$, for $\mathrm{x}_{6}=70$, for $\mathrm{x}_{7}=80$, for $\mathrm{x}_{8}=60$, for $\mathrm{x}_{9}=90$, and for $\mathrm{x}_{10}$ $=20$ respectively. Then the weighted average $\mathrm{a}(\mathrm{X})$ of union fuzzy set $\mathrm{X}$ is “ $\mathbf{0 . 8 5 4}$ ”

Result: Water quality of HOWRAH river is in the grade of "Not Acceptable" with degree of certainty 0.854..

\section{CONCLUSION}

Assessment reveals that quality of river water is not in good book of the PHE Department and needs proper treatment before supplying to local people. The logic of IFS theory has a great capability to give the out put result in more precise numerical form. The model of Fuzzy EIA eliminated the uncertainty from all input basic data upto reasonable extend but it will give more better result if increase the number of study locations of river instead of ten. This Fuzzy EIA model will also help the authority to compare and ranking among all rivers in question of water quality if required for selection of a proposed development project.

\section{REFERENCES}

[1]. Atanassov, K.T.: Intuitionistic Fuzzy Sets, 'Fuzzy Sets and Systems' 20(1986), pp 87-97.

[2]. Biswas. Srijit : Environmental Impact Assessment : An Intuitionistic Fuzzy Approach;

'International Journal of NIFS', Bulgaria. : Vol.4(2), pp.2327 (1998).

[3]. Biswas, Srijit.: A Fuzzy Approach to Environmental Impact Assessment; 'Asian Jour. of Info. Tech', Grace Publications Network- 20054(1), pp35-39 (2005)

[4]. Dungumaro, Esther. W.: Integrated water Resources Management in Tanzania ; AJEAM-RAGEE, Vol-11, pp33-41 (2006).
[5]. Goldman, A. S.; Edwards, A.K. : Integrated Water Resources Planning : National Resources Forum, Vol.16(1), pp 65-70 (1992).

[6]. Green, C. ; Baden, S. ; 'Integrated Water Resources Management : A gender Perspective IDS - Bulletin, Vol.26(1), pp 92-100 (1995).

[7]. Zadeh, L., Fuzzy Sets, Inform. Control., 8(1965), 338353. 